\title{
Bone Mineral Changes and Cardiovascular Effects among Female Athletes with Chronic Menstrual Dysfunction
}

\author{
Ghazaleh Soleimany $^{1}$, MD; Haleh Dadgostar*1,2, MD; Sara Lotfian², MD; \\ Mazyar Moradi-Lakeh ${ }^{3}$, MD; Elham Dadgostar ${ }^{4}$, MD; Shafieh Movaseghi ${ }^{5}$, MD
}

Authors' Affiliation:

1. Department of Sports Medicine, Rasoul Akram Hospital, Tehran University of Medical Sciences, Tehran, Iran

2. Sports Medicine Research Center. Tehran University of Medical Sciences, Tehran, Iran

3. Department of Community Medicine, Tehran University of Medical Sciences, Tehran, Iran

4. Sports Medicine Federation of Islamic Republic of Iran Tehran, Iran

5. Rheumatology Research Center, Tehran University of Medical Sciences, Tehran, Iran

* Corresponding Author;

Address: Sports Medicine Research Center, No.7, Al-e Ahmad Highway, Tehran, Iran

E-mail: hdadgostar@tums.ac.ir

Received: Jun 19, 2011

Accepted: Sep 25, 2011

Key Words: Bone Mineral Density; Cardiovascular Risk Factors; Oligomenorrhea; Female Athletes

\begin{abstract}
Purpose: Oligo/amenorrhea, as a part of the Female Athlete Triad has adverse effects on the athlete's bone mineral density (BMD) and cardiovascular system. Hypoestrogenism, due to suppression of hypothalamus-pituitary axis (HPA) as a result of energy imbalance, is the possible cause of the Triad. This study was designed based on following up and reassessment of elite female athletes who were diagnosed as menstrual dysfunction about two years ago.

Methods: This study was conducted in three phase sections: 1) Reassess the pattern of menstrual cycle among athletes who reported menstrual dysfunction about two years ago; 2) Bone mineral density was measured twice in the same machine and same center with a two-year interval; 3) The laboratory data including blood glucose, lipid profile and inflammatory markers was assessed in phase 3 .

Results: BMD of athletes did not change significantly after 25.5 months of oligomenorrhea $P$ (spine) $=0.2, P$ (femur) $=0.9$. Mean of all cardiovascular factors was in the normal range except for high density lipoprotein (HDL) which was $49.28(\mathrm{SD}=9.18$ ), however, most of the athletes had abnormalities in their lipid profile. Inverse relationship between the increase in the BMD of spine and total cholesterol $(r=-0.49, P=0.04)$, Apolipoprotein A $(r=-0.51$ $P=0.04)$, and very low density lipoprotein (VLDL) $(r=-0.66, P=0.009)$. Also correlation between BMD of spine and HbA1C ( $r=-0.70, P=0.003)$ were significant.

Conclusion: Findings of this study show that negative changes in BMD and cardiovascular biomarkers of female athletes with functional hypothalamic menstrual dysfunction could occur if proper therapeutic intervention (including increase in calorie intake, decrease in exercise load or hormonal replacement) will not consider.
\end{abstract}

Asian Journal of Sports Medicine, Volume 3 (Number 1), March 2012, Pages: 53-58

\section{INTRODUCTION}

$\mathrm{F}$ or the first time in early 1990s, the concept of Female athlete Triad was introduced to describe the interrelated problems of amenorrhea, eating disorders and osteoporosis. Based on a new definition of the Triad, this syndrome is defined as interrelationship among energy availability, menstrual function, and bone mineral density (BMD) in otherwise healthy female athletes ${ }^{[1]}$. According to this classification, low energy availability acts as a primary contributor to disruptions in menstrual function and other complications. Sustained low energy availability, with or without any eating disorder can impair health and cause some medical complications in cardiovascular, endocrine, reproductive, skeletal, gastrointestinal, renal and central nervous systems ${ }^{[1,2]}$. Although many athletes may not be concerned about short term absence of their menses, the lack of estrogen associated with hypothalamus-pituitary axis 
(HPA) suppression can compromise both bone mineral increase during adolescence and young adulthood and also maintenance of bone mineral density later in life. Indeed the other effect of hypoestrogenism seen in amenorrheic athletes is impaired endothelium dependent arterial vasodilation ${ }^{[3]}$, which decreases the perfusion of working muscle, impaired skeletal muscle oxidative metabolism ${ }^{[4]}$ and elevated low-density lipoprotein cholesterol levels ${ }^{[5,6]}$. In other words, similar to low BMD, unfavorable lipid profile, and increased levels of inflammatory markers may be related to menstrual irregularities and estrogen deficiency. Due to the fact that increase of lipid profile with different mechanisms can also be the basis for early cardiovascular disease, athletes with menstrual dysfunction should be assessed more for elevated levels of triglyceride and cholesterol to rule out any predisposing factor of cardiovascular problems.

Even though professional sports for Iranian women has newly come to light, the number of female athletes who have got permission to attend contests wearing Islamic cover in some sport fields at Asian and international levels is increasing. This study is part of a research which was started in 2007. In the first report published by the same researchers in $2009^{\text {[7] }}$, it was shown that $9 \%$ of elite female athletes had been suffering from oligomenorrhea or amenorrhea and this disorder had had a significantly positive relationship with age of under 20 years old, weight class and endurance sports, late onset of menarche and use of oral contraceptives (OCP).

In continuance of this study, to assess the effect of duration on the athletes' health another research was designed. Therefore, this study was aimed to evaluate these athletes' menstrual status and their BMD, lipid profile, and levels of inflammatory markers to find if there is any association between their menstrual irregularities and impairment of these components.

\section{METHODS AND SUBJECTS}

This study is the continuum of a cross sectional research by Dadgostar et al ${ }^{[7]}$ about the menstrual status of all elite Iranian female athletes in 2009. Fiftysix athletes with functional hypothalamic menstrual dysfunction were diagnosed and their BMD was examined. After $25.55(\mathrm{SD}=6.15)$ months, in a new study, they were invited to reassess their bone density change and cardiovascular health status.

Athletes with following reasons were excluded from this study. 1) becoming eumenorrheic in this period of time 2) suffering from chronic diseases, including hyperprolactinemia and thyroid disease; 3) smoker athletes 4) taking any form of hormonal therapy within this time 5) decrease in exercise load and having not performed regular exercise (at least 2 times per week based on reporting from their own federation); 6) history of a clinical diagnosis of polycystic ovarian syndrome.

The study was approved by the ethics committee at Tehran University of Medical Sciences. The ethical standards of the project were in conformity with the provisions of the Declaration of Helsinki of the World Medical Association.

\section{Assessment procedures:}

This study was conducted in three phases. In phase 1 , we invited athletes to come up to the sports medicine clinic of a general hospital. Detailed information about their menstrual pattern, exercise records and medical history were gathered with a questionnaire which was used in previous research ${ }^{[7]}$. Anthropometric findings including weight, height, body mass index (BMI) were also recorded. In order to reassure that the etiology of menstrual dysfunction of our population had not changed from two years ago, all laboratory data, including serum follicle-stimulating hormone (FSH), luteinizing hormone (LH), thyroid-stimulating hormone (TSH), prolactin, free T4, serum testosterone and dehydroepiandrosterone (DHEA), and an ultrasonographic study of the ovary was performed again. From a total of 56 elite female athletes diagnosed as functional hypothalamic menstrual dysfunction, 34 athletes for following reasons were excluded from this study: Persons with increase of energy intake and decrease in exercise load who started to have regular menstruation and/or athletes who used OCPs during this time. A total of 22 athletes were finally evaluated. Secondary amenorrhea was identified 
when menses had ceased at a point in time beyond the establishment of regular menstrual cycles. In this study, secondary amenorrhea is described as the absence for more than three months of the menstrual cycle ${ }^{[8]}$. Oligomenorrhea was defined as menstrual cycles occurring at intervals longer than 35 days ${ }^{[8]}$.

In phase 2, BMD (gram per square centimeter) was measured by dual energy X-ray absorptiometry (DXA) using a Hologic densitometer (Hologic Discover QDR). The measurement parts were the lumbar spine (L1-L4) and femoral neck. The results were expressed in Hologic standards (driven from the NHANSE III study) ${ }^{[9]}$. This assessment was performed by the same machine. Recent guidelines have advocated the use of the term "low BMD" instead of osteopenia in premenstrual syndrome and the use of Z-scores instead of T-scores in young women and children ${ }^{[10]}$. In our study, low BMD was defined as a Z-score between 1.0 and -2.0, and osteoporosis as a Z-score below -2.0. Positive diagnosis was defined as low BMD or osteoporosis at least one of two measurement areas (lumbar spine or neck of femur). The results were compared with the previous bone mineral density which was performed about 25 months ago.

The laboratory data which was measured in phase 3, was divided in three groups; blood glucose, lipid profile and inflammatory markers. Blood glucose was evaluated by measurement of fasting blood glucose (FBS) and hemoglobin $(\mathrm{Hb}) \mathrm{A}_{1} \mathrm{C}$. Lipid profile included total cholesterol, high density lipoprotein (HDL), low density lipoprotein (LDL), very low density lipoprotein (VLDL), triglyceride (TG) and apolipoprotein (Apo) A and Apo B. Fibrinogen and hemocystein took in as inflammatory markers. Beckman CX-3 biochemical autoanalyzer (kit for
HDL, LDL: Pishtaz Teb, for TG , Chol: Parsazmoon, for Apo A, Apo B: Randox.

\section{Statistical analysis:}

Quantitative data were described by mean and standard deviation and qualitative data by frequency. $T$-test and chi-square were used to compare quantitative and qualitative data respectively. The SPSS program (Version 17) was used for all analysis.

\section{RESULTS}

A total of 22 athletes were evaluated. The mean age of participants was $23.55(\mathrm{SD}=5.47)$ (range $=16-35)$. The mean $\mathrm{BMI}$ was $21.66(\mathrm{SD}=2.86 \mathrm{~kg} / \mathrm{m} 2)($ range $=18.21$ - 32.42). Results for low BMD in two measurement areas and their comparison are shown separately in Table 1 and 2.

Based on findings of bone mineral density, two athletes had low BMD ( $Z$ score $<-2)$ in femural neck and four athletes showed low BMD in their spinal region, as the $Z$ score of three of them was between -1 and -2 and one of them demonstrate Z-score $<-2$. Both BMD of spinal region and femoral neck less than -2 , belonged to the same athlete.

Table 3 shows cardiovascular biomarkers, which were measured once after two years of documented oligo/amenorrhea.

Our data also revealed that the prevalence of high levels of total cholesterol; LDL and TG were $15.78 \%$ (3/19). HDL lower than 50 was $63.15 \%(12 / 19)$ and

Table 1: Distribution of bone mineral density in spine and femural neck

\begin{tabular}{|c|c|c|}
\hline \multicolumn{2}{|c|}{ Bone mineral density } & Number (Percentage) \\
\hline \multirow{4}{*}{ Spine } & $\mathrm{z}$ score $>-1$ & $17(77.3 \%)$ \\
\hline & $\mathrm{z}$ score; -1 to -2 & $4(18.2 \%)$ \\
\hline & $\mathrm{z}$ score $<-2$ & $1(4.5 \%)$ \\
\hline & Total & $22(100 \%)$ \\
\hline \multirow{4}{*}{ Femur } & $\mathrm{z}$ score $>-1$ & $19(86.4 \%)$ \\
\hline & $\mathrm{z}$ score $;-1$ to -2 & $1(4.5 \%)$ \\
\hline & z score $<-2$ & $2(9.1 \%)$ \\
\hline & Total & $22(100 \%)$ \\
\hline
\end{tabular}


Table 2: Comparison mean values of BMD in two separate regions between two years

\begin{tabular}{lccc} 
Region of BMD & $\begin{array}{c}\text { At the beginning } \\
\mathbf{N}=\mathbf{2 2}\end{array}$ & $\begin{array}{c}\text { After } \mathbf{2 5} \text { months } \\
\mathbf{N}=\mathbf{2 2}\end{array}$ & P value \\
\hline Densitometry of spine & $1.002(0.117)$ & $1.012(0.120)$ & 0.2 \\
Densitometry of femur neck & $0.887(0.127)$ & $0.878(0.117)$ & 0.9
\end{tabular}

BMD: Bone Mineral Density

low Apo $\mathrm{A}$ and $\mathrm{HbA}_{1} \mathrm{C}$ more than 6 were $55.55 \%$ $(10 / 18)$ and $31.25 \%(5 / 14)$, respectively. It should be mentioned that an $\mathrm{HbAlC}$ level more than 7 was

Our data also revealed that the prevalence of high levels of total cholesterol; LDL and TG was $15.78 \%$ (3/19). HDL lower than 50 was $63.15 \%(12 / 19)$ and, low Apo $\mathrm{A}$ and $\mathrm{HbA}_{1} \mathrm{C}$ more than 6 were $55.55 \%$ $(10 / 18)$ and $31.25 \%(5 / 14)$, respectively. It should be mentioned that an $\mathrm{HbA1C}$ level more than 7 was $12.5 \%(2 / 16)$.

In athletes with menstrual disturbance, we found inverse correlation between the rate of increase in bone mineral density and total cholesterol $(\mathrm{r}=-0.49 P=$ $0.04)$, Apo A ( $r=-0.51, P=0.03)$, and VLDL $(r=-0.66$, $P=0.009)$. Correlation between the rate of increase in bone mineral density and blood lipid levels including LDL, TG, HDL, Apo B, and FBS was not significant. However, $\mathrm{HbA} 1 \mathrm{C}$ was inversely correlated with the rate of increase in bone mineral density $(r=-0.70$, $P=0.003)$.

\section{DISCUSSION}

The design of this study was based on the follow-up of elite female athletes who were diagnosed with menstrual dysfunction about two years ago. We decided to reassess their menstrual pattern and find out if the prolongation of the menstrual dysfunction can affect their cardiovascular biomarkers and/or change their previous BMD which was measured about two years ago. To the best of our knowledge, this is the first study to evaluate alteration of BMD and its association with cardiovascular biomarkers among an Iranian elite female athletes group with menstrual dysfunction. Findings of this study show that changes in BMD and cardiovascular biomarkers of female athletes with functional hypothalamic menstrual dysfunction could occur if proper therapeutic intervention (including increase in calorie intake, decrease in exercise load or hormonal replacement) are not considered. We also found an inverse relationship between the rate of increase in bone mineral density of spine region and

Table 3: Mean (SD) of Blood glucose, serum lipids and inflammatory biomarkers in female athletes with menstrual dysfunction

\begin{tabular}{|c|c|c|c|}
\hline Variables & $\begin{array}{c}\text { Low BMD } \\
(n=4)\end{array}$ & $\begin{array}{c}\text { Normal BMD } \\
(n=14)\end{array}$ & Normal range (mg/dl) \\
\hline Fasting blood sugar & $87.33(10.26)$ & $88.30(6.97)$ & $70-115 \mathrm{mg} / \mathrm{dl}$ \\
\hline Hemoglobin $A_{1} C$ & $5.63(1.27)$ & $5.38(0.98)$ & $4-6 \%$ \\
\hline Total cholesterol & $193.00(52.64)$ & $166.80(30.73)$ & $\mathrm{Up}$ to $200 \mathrm{mg} / \mathrm{dl}$ \\
\hline Triglyceride & $100.75(86.93)$ & $78.80(36.46)$ & $\mathrm{Up}$ to $150 \mathrm{mg} / \mathrm{dl}$ \\
\hline Low density lipoprotein & $83.75(22.16)$ & $78.07(17.88)$ & Up to $130 \mathrm{mg} / \mathrm{dl}$ \\
\hline Very low density lipoprotein & $44.67(33.08)$ & $39.33(16.98)$ & Up to $40 \mathrm{mg} / \mathrm{dl}$ \\
\hline High density lipoprotein & $51.00(12.06)$ & $49.53(8.86)$ & $\geq 35 \mathrm{mg} / \mathrm{dl}$ \\
\hline Apolipoprotein A & $155.50(53.51)$ & $125.42(29.14)$ & $120-176 \mathrm{mg} / \mathrm{dl}$ \\
\hline Apolipoprotein B & $96.00(10.80)$ & $70.17(15.58)$ & $63-114 \mathrm{mg} / \mathrm{dl}$ \\
\hline Homocysteine & $9.95(1.48)$ & $11.02(2.98)$ & 5- $15 \mu \mathrm{mol} / \mathrm{L}$ \\
\hline Fibrinogen & $274.00(132.94)$ & $215.79(35.98)$ & $200-400 / \mathrm{dl}$ \\
\hline
\end{tabular}

SD: Standard Deviation / BMD: Bone Mineral Density 
total cholesterol and VLDL levels of elite female athletes. Although physical activity can maintain and increase BMD, the important question asked by a health professional is if this generalization can be applied to high risk female athletes or not ${ }^{[11]}$ ?

The mechanism for low BMD and an impaired lipid profile in athletes with menstrual dysfunction is probably related to hypoestrogenism. Previous studies have shown that bone mass is affected by the length and severity of menstrual dysfunction ${ }^{[12]}$. Due to the fact that the peak bone mass gain in a human being occurs during the second and third decade of his/ her life, lower bone density in athletes with functional hypothalamic menstrual dysfunction may predispose them to some complications like stress fracture and/or osteoporosis which can disturb their function. Moreover, it has previously been demonstrated that increased levels of lipid profile such as total cholesterol, LDL and VLDL are associated with hypogonadal states, such as in anorexia nervosa and athletic amenorrhea ${ }^{[13,14]}$.

Our finding is consistent with the finding of several studies whose authors ${ }^{[15,16]}$ have reported nonsignificant increases in BMD of athletes who have not taken an appropriate therapy. The prevalence of low BMD in healthy young females has not been broadly studied and there are limited normative databases to offer reference norms for this age group ${ }^{[11]}$. In a study reported by Cobb et al, athletes ${ }^{[17]}$ with persistent menstrual dysfunction who did not receive OCs, did not have any change in their bone mass, which is in agreement with our study. Gibson et al, also found that female athletes with menstrual dysfunction after 9-12 months showed significantly lower BMD than the eumenoric group ${ }^{[18]}$. Due to the fact that the etiology of bone loss is multifactorial, measurement of bone density can be helpful to convince high risk athletes into intervention in terms of increasing nutritional intake, decreasing intensity of exercise or prescribing hormone replacement therapy.

Elevated lipid levels may be reported in premenstrual women with hypoestrogenic conditions secondary to caloric deficiency such as anorexia nervosa ${ }^{[13,14]}$. Based on findings by Friday et al ${ }^{[19]}$, increased level of LDL profiles in amenorrheic athletes comparing with eumenorrheic athletes have been described. Consistent with these studies, our finding shows inverse relationship between the rate of increase in bone mineral density and total cholesterol and VLDL levels of elite female athletes. The main cause of amenorrhea in athletes seems to be an inhibition of the hypothalamus-pituitary-ovarian axis and it might be that prolonged exercise-induced amenorrhea has adverse cardiovascular consequences ${ }^{[20,21]}$. As a rule, we consider a physically active lifestyle to be defending against the development of atherosclerosis. Although the amenorrheic athletes are hypoestrogenic, the range of their age, physical activity and also their health condition usually protects them from cardiovascular disease.

Unlike authors of some studies ${ }^{[22,23]}$ we not only did not find any relationship between alteration of BMD and serum level of LDL, but also there was an inversely correlated with the rate of increase in bone mineral density and serum concentration of Apo A. Although estrogen is known to decrease low-density lipoprotein (LDL) oxidation ${ }^{[22]}$, which are critical steps in the progression to atherosclerosis, it has been shown that apolipoproteins are a more important risk factor to predispose patients to coronary disease ${ }^{[24]}$ and therefore should be included in the lipid analysis. However, this finding may be a result of the small number of athletes who were evaluated based on their menstrual dysfunction history. It should be mentioned that, we only assessed the female athletes with clinical menstrual dysfunction, and based on the new definition of the Triad ${ }^{[1]}$ athletes with subclinical menstrual dysfunction were missed. Therefore we may underestimate the prevalence of menstrual dysfunction with its complications.

Despite the fact that there is not any significant correlation of FBS and alteration of BMD in our study, $\mathrm{HbA}_{1} \mathrm{C}$ was inversely correlated with the rate of increase in bone mineral density. This inconsistency may be due to the small number of our subjects.

Limitation in our study should be noted. Firstly, one of the limitations of the study was the small number of athletes who were evaluated. Because this research was designed on the basis of following up of elite female athletes with history of clinical menstrual dysfunction about two years ago, our study population was limited. Therefore we could not add any new 
patient to increase our sample size. Another limitation is the absence of normal athletes as control group to in the study. Thirdly, because we cannot supervise on the nutritional diet and intensity of exercise of athleteswith different types of sports-during the study, prediction of their energy availability cannot occurr. Therefore, their low energy availability as a primary contributor to disruptions in menstrual function and bone mass was not assessed.

\section{CONCLUSION}

Based on our finding, there is an inverse relation between the rate of increase in bone mineral density of spine region and total cholesterol and VLDL levels. This result shows that each factor negatively affects bone mineral density, that can also impair lipid profile. Therefore, chronic hypoestrogenism could be a risk factor of cardiovascular disease for adult female athletes despite their regular physical activities.
However, we recommend that any future study should use a better method to identify cases of low energy availability and subclinical menstrual disorder to find the high risk of athletes earlier. In addition, to find the clinical inferences of impaired levels of lipid profile among elite female athletes with functional hypothalamic menstrual dysfunction, longitudinal studies in this group of women should be designed. In addition, clinical trial studies should be created to find if a high serum concentration of lipid profile is reversible after treatment of menstrual dysfunction. Moreover, the long-term effects of this condition for future risk of cardiovascular diseases are unknown.

\section{ACKNOWLEDGMENTS}

This study is part of a post graduated dissertation supported by Tehran University of Medical sciences.

\section{Conflict of interests: None}

\section{REFERENCES}

1. Nattiv A, Loucks AB, Manore MM, et al. American College of Sports Medicine position stand. The female athlete triad. Med Sci Sports Exerc 2007;39:1867-82.

2. Golden NH. The adolescent: vulnerable to develop an eating disorder and at high risk for long-term sequelae. Ann N Y Acad Sci 1997; 817:94-7.

3. Hoch AZ, Lal S, Jurva JW, Gutterman DD. The female athlete triad and cardiovascular dysfunction. Phys Med Rehabil Clin N Am 2007;18:385-400.

4. Hergenroeder AC, Smith EO, Shypailo R, et al. Bone mineral changes in young women with hypothalamic amenorrhea treated with oral contraceptives, medroxyprogesterone, or placebo over 12 months. Am J Obstet Gynecol 1997;176: 1017-25.

5. Friday KE, Drinkwater BL, Bruemmer B, et al. Elevated plasma low-density lipoprotein and high-density lipoprotein cholesterol levels in amenorrheic athletes: effects of endogenous hormone status and nutrient intake. J Clin Endocrinol Metab 1993;77:1605-9.

6. O'Donnell E, De Souza MJ. The cardiovascular effects of chronic hypoestrogenism in amenorrhoeic athletes: a critical review. Sports Med 2004;34:601-27.

7. Dadgostar H, Razi M, Aleyasin A, et al. The relation between athletic sports and prevalence of amenorrhea and oligomenorrhea in Iranian female athletes. Sports Med Arthrosc Rehabil Ther Technol 2009;1:16.

8. Diaz A, Laufer MR, Breech LL. Menstruation in girls and adolescents using the menstrual cycle as a vital sign. Pediatrics 2006; $118: 2245-50$

9. Looker AC, Wahner HW, Dunn WL, et al. Updated data on proximal femur bone mineral levels of US adults. Osteoporos Int 1998;8:468-89

10. Leib ES, Lewiecki EM, Binkley N, Hamdy RC. Official positions of the International Society for Clinical Densitometry. South Med J 2004;97:107-10.

11. Bernadette L Matthews, Kim L Bennell. Bone Health and Young Females. Journal of women Health physical Therapy 2005;29:3:1927. 
12. Dequeker J, Nijs J, Verstraeten A, et al. Genetic determinants of bone mineral content at the spine and radius: a twin study. Bone $1987 ; 8: 207-9$

13. Case T, Lemieux S, Kennedy SH, Lewis GF. Elevated plasma lipids in patients with binge eating disorders are found only in those who are anorexic. Int J Eat Disord 1999;25:187-93.

14. Feillet F, Feillet-Coudray C, Bard JM, Parra HJ, Favre E, Kabuth B, et al. Plasma cholesterol and endogenous cholesterol synthesis during refeeding in anorexia nervosa. Clin Chim Acta 2000;294:45-56.

15. Cumming DC. Exercise-associated amenorrhea, low bone density, and estrogen replacement therapy. Arch Intern Med 1996;156: 21935 .

16. Gremion G, Rizzoli R, Slosman D, et al. Oligo-amenorrheic long-distance runners may lose more bone in spine than in femur. Med Sci Sports Exerc 2001;33:15-21

17. Cobb KL, Bachrach LK, Sowers M, et al. The effect of oral contraceptives on bone mass and stress fractures in female runners. Med Sci Sports Exerc 2007;39:1464-73.

18. Gibson JH, Mitchell A, Reeve J, Harries MG. Treatment of reduced bone mineral density in athletic amenorrhea: a pilot study. Osteoporos Int 1999;10:284-9.

19. Friday KE, Drinkwater BL, Bruemmer B, et al. Elevated plasma low-density lipoprotein and high-density lipoprotein cholesterol levels in amenorrheic athletes: effects of endogenous hormone status and nutrient intake. J Clin Endocrinol Metab 1993;77:1605-9.

20. Rickenlund A, Thoren M, Nybacka A, et al. Effects of oral contraceptives on diurnal profiles of insulin, insulin-like growth factor binding protein-1, growth hormone and cortisol in endurance athletes with menstrual disturbance. Hum Reprod 2010;25:85-93.

21. Loucks AB, Thuma JR. Luteinizing hormone pulsatility is disrupted at a threshold of energy availability in regularly menstruating women. J Clin Endocrinol Metab 2003;88:297-311.

22. Cid MC, Schnaper HW, Kleinman HK. Estrogens and the vascular endothelium. Ann N Y Acad Sci 2002;966:143-57.

23. Mendelsohn ME, Karas RH. The protective effects of estrogen on the cardiovascular system. N Engl J Med 1999;340:1801-11.

24. Walldius G, Jungner I. Apolipoprotein B and apolipoprotein A-I: risk indicators of coronary heart disease and targets for lipidmodifying therapy. J Intern Med 2004;255:188-205. 